\title{
Investigation of the molecular characteristics of Brucella isolates from Guangxi Province, China
}

\author{
Zhi-guo Liu' ${ }^{1,2}$, Miao Wang ${ }^{3}$, Hong-yan Zhao ${ }^{1}$, Dong-ri Piao ${ }^{1}$, Hai Jiang ${ }^{1}$ and Zhen-jun Li*
}

\begin{abstract}
Background: Human brucellosis has become a severe public health problem in China's Guangxi Province, and there has been higher prevalence of brucellosis in this region after 2010. Both multiple locus variable-number tandem repeat analysis (MLVA) and multilocus sequence typing (MLST) assay schedules were used to genotype isolates and determine relationships among isolates.
\end{abstract}

Results: A total of 40 isolates of Brucella were obtained from humans, pigs, and dogs from 1961 to 2016. There were at least three species of Brucella detected in Guangxi Province, Brucella melitensis, Brucella suis, and Brucella canis, with 16, 17, and 7 isolates, respectively. Of which B. suis biovar 3 was the predominant species resulting in pig brucellosis in the area examined before 2000s. Moreover, B. melitensis biovar 3 was found to be mainly responsible for human brucellosis during 2012-2016. All B. melitensis isolates in this study belonged to East Mediterranean lineage. MLVA-11 genotype 116 was the dominant genotype and represented $81.2 \%$ of the isolates. MLVA cluster analysis showed there to be $44 \%(7 / 16)$ brucellosis cases caused by B. melitensis with a profile of outbreak epidemic from 2012 to 2016. However, nearly 83.3\% (20/24) of brucellosis cases resulting from both B. suis and B. canis showed no epidemiological links or sporadic characteristics. MLVA-16 analysis confirmed extensive genotypesharing events between B. melitensis isolates from Guangxi and other northern provinces within China. These data revealed that there are potential epidemiology links among these strains. B. suis strains of this study showed a unique genetic lineage at the global level and may have existed historically in this area. However, present B. canis isolates were closely related to previously reported isolates in Korea, where they may have originated. MLST typing showed that the population structure of Brucella strains had changed considerably in this province; ST17 and ST21, two previously predominant populations appeared to have been replaced by recently emerging ST8 group.

Conclusions: Our investigation data have inspired the hypothesis that Guangxi Province had been subject to an imported human brucellosis epidemic. Our data suggest that strains found in Northern regions of China are the principal source of infections in recent cases of human brucellosis in Guangxi Province. Comparative genomic analysis from more strains is necessary to confirm this hypothesis. This work will facilitate better understanding of the epidemiology and improve the effectiveness of control and prevention of brucellosis in this region.

Keywords: B. melitensis, B. suis, MLVA, MLST, Molecular characteristics, Guangxi

\footnotetext{
* Correspondence: lizhenjun@icdc.cn

'State Key Laboratory for Infectious Diseases Prevention and Control,

National Institute for Communicable Disease Control and Prevention,

Chinese Center for Disease Control and Prevention, 155 Changbai Road,

Changping, Beijing 102206, People's Republic of China

Full list of author information is available at the end of the article
}

(c) The Author(s). 2019 Open Access This article is distributed under the terms of the Creative Commons Attribution 4.0 International License (http://creativecommons.org/licenses/by/4.0/), which permits unrestricted use, distribution, and reproduction in any medium, provided you give appropriate credit to the original author(s) and the source, provide a link to the Creative Commons license, and indicate if changes were made. The Creative Commons Public Domain Dedication waiver (http://creativecommons.org/publicdomain/zero/1.0/) applies to the data made available in this article, unless otherwise stated. 


\section{Background}

Brucellosis is a common zoonotic disease caused by the genus Brucella, a Gram-negative, facultative intracellular bacteria that infects a wide range of mammals, including domestic and wild animals as well as humans [1, 2]. Human brucellosis is largely dependent on the animal reservoirs and spreads through direct contact with infected animals or consumption of contaminated animal products [3]. B. melitensis, B. abortus, and $B$. suis are highly pathogenic and a frequent causative pathogen of animal and human brucellosis [4]. B. melitensis is the predominant circulating strain in northeastern and northwestern China and it produces the most severe infections in humans [5]. $B$. abortus is the main etiological agent of brucellosis in cattle, but it is associated with far fewer cases of human disease $[6,7]$. Human cases due to $B$. abortus are sporadic in China and, in Sichuan, B. abortus is the predominant strain isolated. It generally causes infections less severe than those caused by $B$. melitensis or B. suis $[8,9]$. B. suis is the most disseminated species in south regions of China and are associated with sporadic brucellosis epidemics [10]. Recently, due to unrestricted movements of a large number of infected small ruminants and other illegal trade, the geographic distribution profiles of Brucella species and transmission patterns have changed [11]. The identification and molecular typing of the circulate Brucella species is extremely essential for epidemiological follow-up and control of the disease [12]. Classical biotyping is the gold standard for investigations of phenotypic characteristics, and deeper molecular epidemiological investigations can be used to trace back the source of infection to its geographic origin and determine relationships among isolates [13].

Guangxi Province was the site of an historic brucellosis epidemic; B. suis predominates in this region [9]. The first case of brucellosis was reported in 1952 and found to have been caused by imported infected pigs from other regions. Some B. suis strains were later isolated [14]. Human brucellosis is limited to few cases every year and its incidence was low before 1990. Most of these cases of brucellosis were caused by B. suis and $B$. canis. Since 2012, the number of human brucellosis cases has increased every year [15]. However, molecular typing and epidemiology characteristics of Brucella isolates from Guangxi remain unknown. In this report, we present the results of a study performed by Brucella MLVA16 assay on 40 human and animal field isolates of B. melitensis, B. suis, and B. canis. The aims of this study were to investigate causes of the increase in the incidence of human brucellosis and to estimate the epidemiological relationship of isolates for a trace-back survey of the source of infections in Guangxi, China.

\section{Methods}

\section{Bacterial strains and DNA preparation}

A total of 40 isolates were isolated from Guangxi province between 1961 and 2016 year. They represent all Brucella isolates collected during this period in Guangxi province. These strains were isolated from human blood, dog and pig at the first line laboratory by the Guangxi Center for Infectious Disease Control and Prevention. 18 were recovered from human blood, 15 from pig, and 6 from dog. B. melitensis bv. $116 \mathrm{M}$ was used as control strain to calibrate the VNTR units. DNA was isolated using Full-automatic nucleic acid extraction system (LLXBIO China Ltd., China) extraction from 48-h cultures according to the manufacturer's instructions. DNA extracted from all isolates was stored at $-20^{\circ} \mathrm{C}$.

\section{Brucella biotyping}

All isolates were identified as Brucella species on the basis of morphology and conventional identification methods according to standard biotyping procedures, including requirement of $\mathrm{CO}_{2}$ for growth, $\mathrm{H}_{2} \mathrm{~S}$ production, sensitivity to thionin (10 and $20 \mu \mathrm{g} / \mathrm{ml}$ ), basic fuchsin $(20 \mu \mathrm{g} / \mathrm{ml})$ and agglutination with mono-specific antiserum for $\mathrm{A}$ and $\mathrm{M}$ antigens and phage lysis test (Tbilisi, Tb; Berkeley, Bk2; Weybridge; Wb) [16, 17]. Reagents and pages were obtained from the National Institute for Communicable Disease Control and Prevention, which is China's center for infectious disease control and prevention. B. melitensis $16 \mathrm{M}(\mathrm{BM})$ and B. abortus 544 (BA) and B. suis 1330 (BS) were used as control strains.

\section{MLVA-16 genotyping}

All of isolates were further examined by MLVA, genotyping schedule, PCR amplification process as described previously $[13,18]$. PCR products were preliminarily evaluated by $2 \%$ or $3 \%$ agarose gel electrophoresis. Then, positive products were denatured and resolved by capillary electrophoresis on an ABI Prism 3130 automated fluorescent capillary DNA sequencer (Applied Biosystems). Fragments were sized following comparison with a ROX (carboxy-X-rhodamine)-labeled molecular ladder (MapMaker 1000; Bioventures Inc., Murfreesboro, TN, USA) and Gene Mapper software version 4.0 (Applied Biosystems). The fragment sizes were converted to repeat unit numbers using a published allele numbering system [19].

\section{MLST genotyping}

MLST genotyping was performed by analyzing nine distinct genomic locus, including seven housekeeping genes (gap, aroA, glk, dnaK, gyrB, trpE, and cobQ), one outer membrane protein (omp25), and one intergenic fragment (int-hyp) [20]. PCR amplification was performed as described previously [21]. Sequences obtained from 
purified PCR products were aligned using MEGA 6.0 software according to published MLST sequences in GenBank (accession numbers AM694191-AM695630) [20]. A local comparison database was established after downloading of relevant data, and distinct alleles identified at the nine selected loci were each given a numerical designation according to the sequences of the defined alleles. Each sequence type over all loci (ST) was predicted by comparisons and analyses based on a local comparison database established using MEGA 6.0 and a webbased MLST service (Brucella Base, https://pubmlst.org/ brucella/). DNA preparations from the $B$. melitensis 16 M, B. abortus 544, and B. suis 1330 reference strains were used as controls.

\section{Analysis of genotyping data}

MLVA data were analyzed using BioNumerics version 7.6 software (Applied Maths, Belgium). Both categorical coefficient and un-weighted pair group methods with arithmetic mean algorithm (UPGMA) were applied to MLVA clustering analysis (Additional file 1: Table S1). Resultant genotypes were compared using the online Brucella 2016 MLVAbank. The MLVA-11 characters (combination of panels 1 and 2A loci) of the isolates were compared to those of strains in the 2016 MLVA bank to determine the geographic origin of each strain; minimum spanning tree (MST) based on complete MLVA-16 was used to investigation molecular relationships between strains in this study and 340 isolates including three species: $B$. melitensis $(n=296), B$. suis $(n=$ $15)$, and B. canis $(n=29)$ from other provinces of China (MLVAbank_V1.4.0) (Additional file 2: Table S2). MLVA-16 was used for genetic relationship investigation of both Brucella suis and canis on a global scale $(n=$ 615) (Additional file 3: Table S3). The resulting MLST genotypes were compared using the web-based MLST database (https://pubmlst.org/brucella/) (Additional file
4: Table S4). MLST dendrogram was constructed by BioNumerics version 7.6 software. MLVA and MLST profiles of 40 isolates have been submitted to the MLVA bank_V1.4.0 (http://microbesgenotyping. i2bc. parissaclay.fr/).

\section{Results}

\section{Characteristics and distributions of isolates}

All strains from this study exhibited a convex, circular, and translucent morphology profile. A slight blue color was observed under sunlight and gram-negative coccobacilli were observed, resembling fine sand under the microscope. The growth characteristics, phage lysis experiments, dye bacteriostatic tests, and slide agglutination with monospecific anti-Brucella sera were used to characterize all isolates (Table 1). Species and biovar were discriminated based on standard bacteriological procedures. Finally, biotyping identified 16 strains as $B$. melitensis biovar 3, 18 strains as B. suis (biovar $1(n=2)$ and biovar $3(n=15))$, and 7 as B. canis. 19 strains were isolated from human blood, 21 out of 40 from animals (19 from pigs and 6 from dogs) (Table 2). Geographic distribution profiles of some strains were unknown (according to requests made by the local department of health, it is not permitted to publish the locations of $B$. melitensis isolates).

\section{MLVA typing of isolates}

Using panel 1 markers, the present population clustered into nine genotypes: $42(1-5-3-13-2-2-3-2 ; N=14), 114$ (1-5-3-13-3-2-3-2; $N=1), 58(1-5-3-13-3-1-3-2 ; N=$ 1), N1 (2-3-3- $11-2-1-5-6 ; N=12), \mathrm{N} 2(2-3-3-11-3-1-$ $5-6 ; N=4)$, N3 (1-3-3-11-2-1-5-6; $N=1)$, N4 (2-3-111-2- 1-5-7; $N=1)$, N5 (2-3-3-11-2-1-5-7; $N=5)$ and N6 (2-3-3-11-3-1-5-7; N=1). Clustering analysis showed that the 40 isolates formed three main clusters (A-C) (Fig. 1). Cluster A had three genotypes (42, 114

Table 1 Biotyping characteristics of Brucella species isolates in Guangxi, China

\begin{tabular}{|c|c|c|c|c|c|c|c|c|c|c|c|}
\hline \multirow[t]{2}{*}{ Strain No. } & \multicolumn{4}{|c|}{ Growth characteristics } & \multicolumn{3}{|c|}{ Monospecific Sera } & \multicolumn{3}{|c|}{ Phages lysis testing } & \multirow[t]{2}{*}{ Interpreted } \\
\hline & $\mathrm{CO}_{2}$ requested & $\mathrm{H}_{2} \mathrm{~S}$ & $\mathrm{BF}$ & $\mathrm{TH}$ & A & M & $\mathrm{R}$ & $\mathrm{Tb}$ & $\mathrm{BK}_{2}$ & $\mathrm{~Wb}$ & \\
\hline $\mathrm{BA}$ & + & + & + & - & + & - & - & + & + & + & B. abortus 544 \\
\hline $\mathrm{BM}$ & - & - & + & + & - & + & - & - & + & & B. melitensis $16 M$ \\
\hline BS & - & ++ & - & + & + & - & - & - & + & + & B. suis 1330 \\
\hline GX001 015, GX040 & - & - & + & + & + & + & - & $\mathrm{NL}$ & $\mathrm{CL}$ & $\mathrm{NL}$ & B. melitensis bv. 3 \\
\hline GX021, GX024 & - & ++ & - & + & + & - & - & $\mathrm{NL}$ & $\mathrm{CL}$ & $\mathrm{CL}$ & B. suis bv. 1 \\
\hline GX016 021, 022, 023, GX025 032 & - & - & + & + & + & - & - & $\mathrm{NL}$ & $C L$ & $\mathrm{CL}$ & B. suis bv. 3 \\
\hline GX033 GX039 & - & - & - & + & - & - & + & $\mathrm{NL}$ & $\mathrm{NL}$ & $\mathrm{NL}$ & B. canis \\
\hline
\end{tabular}

Strain No., the number conferred to isolates;

BF, Basic fuchsin at $20 \mu \mathrm{g} / \mathrm{ml}(1 / 50,000 \mathrm{w} / \mathrm{v}) ; \mathrm{TH}$, Thionin at $20 \mu \mathrm{g} / \mathrm{ml}(1 / 50,000 \mathrm{w} / \mathrm{v})$;

Phages, $\mathrm{Tb}=$ Tbilisi, $\mathrm{BK}_{2}=$ Berkeley type $2, \mathrm{~Wb}=$ Weybridge;

$\mathrm{CL}$, Confluent Lysis; NL, No lysis; RTD, Routine test dilution;

+ , positive (serum agglutination positive);

-, negative (serum agglutination negative) 
Table 2 Host, sample ID, and sample size distribution of Brucella species in Guangxi, China

\begin{tabular}{clll}
\hline Location & Host & Sample ID & Sample size \\
\hline Guangxi & Human & GX001-015, GX018-020, GX040 & 19 \\
& Pig & GX016, GX017, GX021-032, GX036 & 15 \\
& Dog & GX033-036, GX037-039 & 6
\end{tabular}

Location, the regions of sample collection

Host, the hosts from which the bacteria were isolated

Sample ID: serial number for the 40 isolates

and 58); cluster B had three new genotypes (N4-N6), cluster C had also three new genotypes (N1 - N3). Using the Brucella 2016 MLVA database and panel 1 markers, found samples contained three species: B. melitensis (genotypes 42,58 , and 114 ; cluster A), genotypes 42 represent $87.5 \%(14 / 16)$ of isolates and plays a dominant role in B. melitensis. B. suis (genotype N1-N3; cluster C and B) and B. canis (genotype N4-N6; cluster B).

MST characterization of isolates based on MLVAs analysis Based on previously studies, all B. melitensis isolates belonged to East Mediterranean lineage, MLVA-11 genotypes 116 represent $81.2 \%$ of strains. In contrast, $B$. suis and B. canis yielded three novelty MLVA-11 genotypes, respectively; compared with correspond strains from other regions in 2016 MLVAbank. A Minimum Spanning Tree based on MLVA-16 analysis showed that there were five shared genotypes observed among seven $B$. melitensis strains in this study and other regions in China; the remaining nine $B$. melitensis were very close to strains from other provinces. B. suis and B. canis from this study represent unique genetic lineage and formed a relative independence branch, which is divergence obviously with strains from China (Fig. 2). In order to determine the origin and assess the genetic relationships of $B$. suis and B. canis from this study, we analyzed MST among strains collected from many parts of the globe. This analysis showed that $B$. suis strains had unique genetic lineages at the global level. However, $B$. canis isolates were very similar to strains from Korea (Fig. 3).

\section{Cluster analysis for Guangxi Province Brucella strains genotypes}

A total 34 genotypes were yielded using complete MLVA-16 schedule. Cluster B and C including 6 panel 1

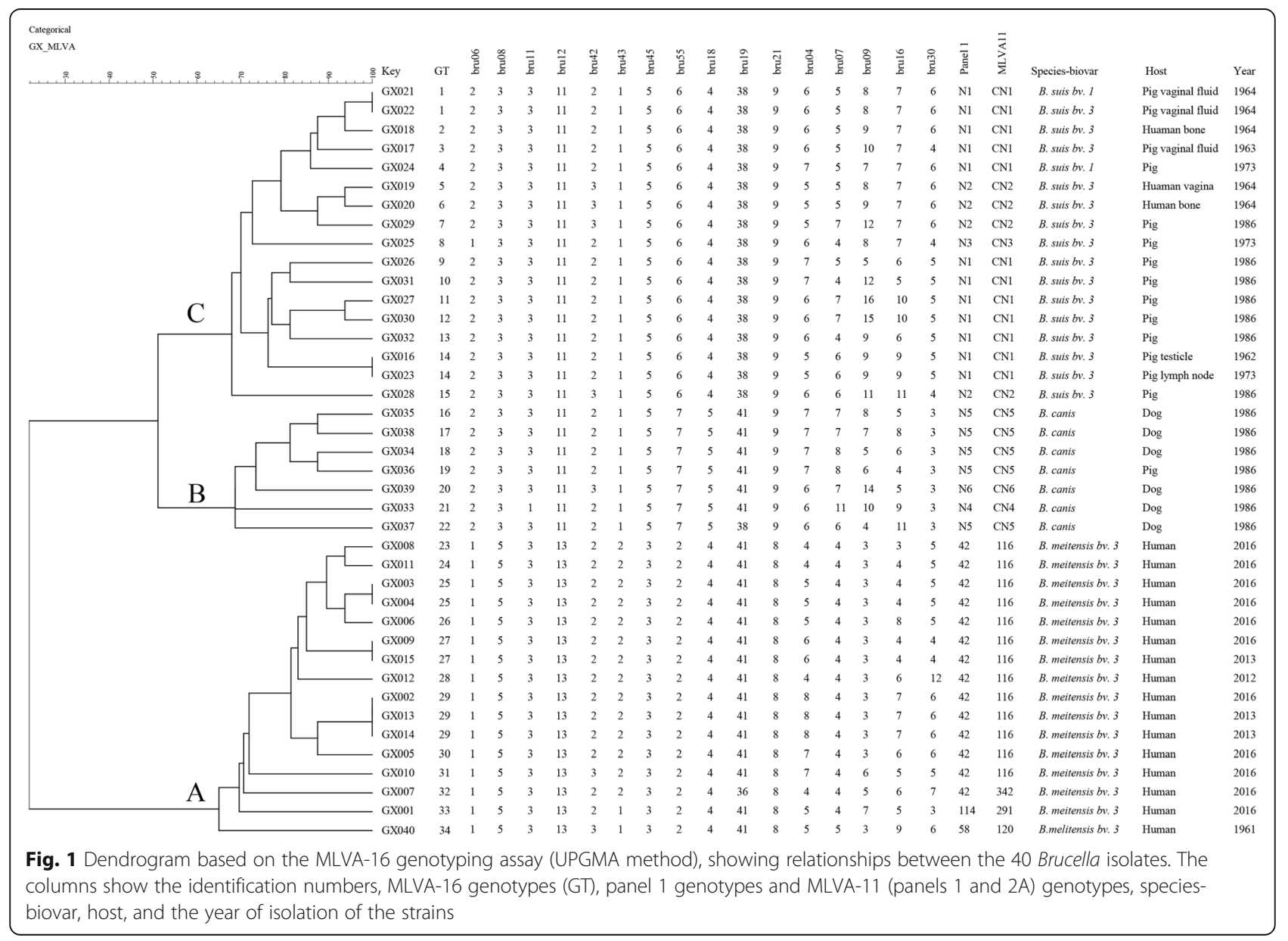




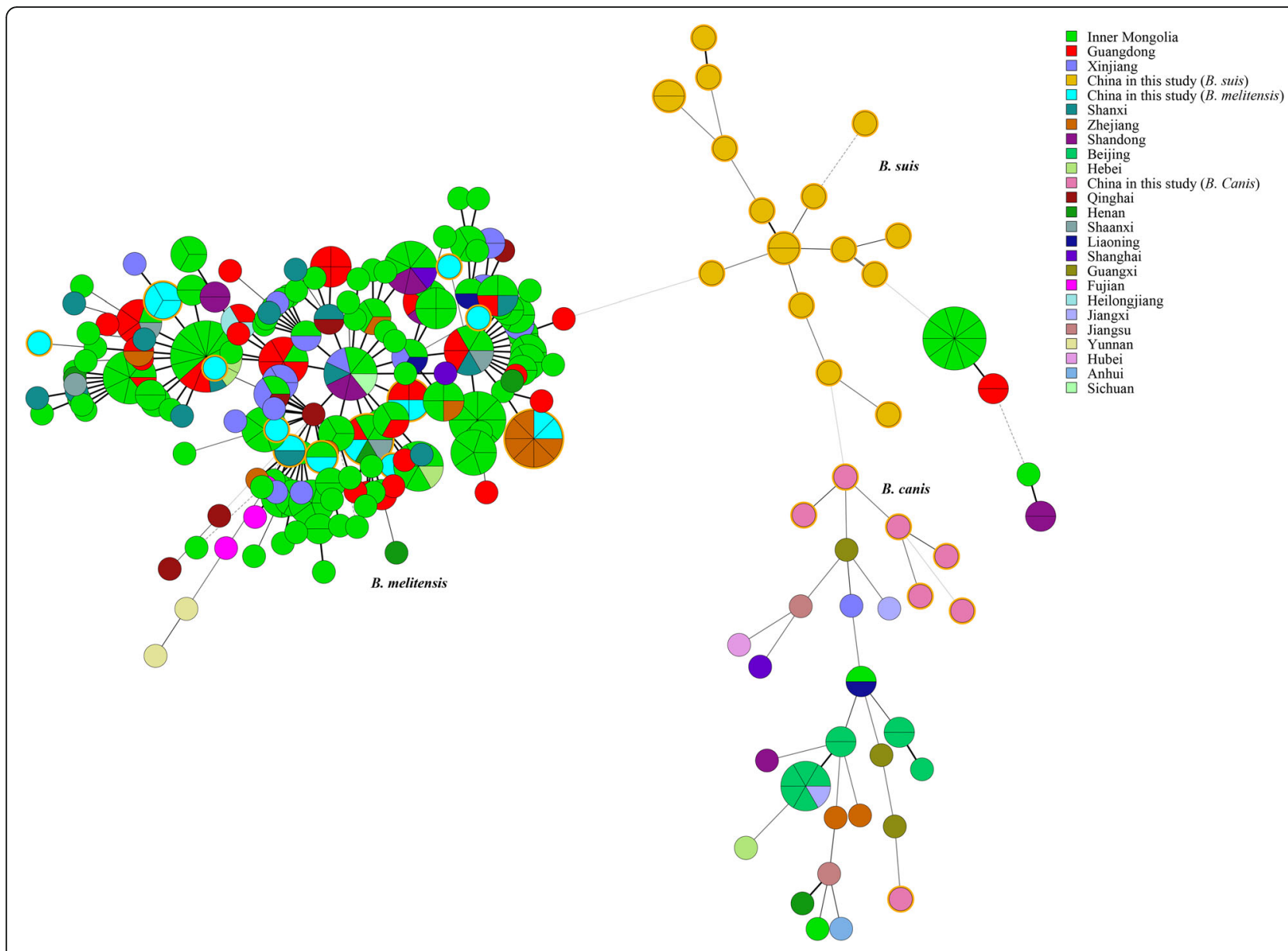

Fig. 2 Minimum spanning tree based on MLVA-16 data for Brucella strains in China wide

genotypes and comprised by 24 Brucella strains were isolated before 2010, and which of four strains were shared two identical MLVA-16 genotypes, respectively; other twenty strains each represent single MLVA-16 genotypes. Cluster A incorporating three panel 1 genotypes and comprised of 15 Brucella strains was isolated after 2010. Three MLVA-16 genotypes were shared by two to three strains, and the cluster rate was $44 \%(7 / 16)$. The remaining nine strains each represent single MLVA-16 genotypes.

Molecular epidemiological investigation of 296 Chinese $B$. melitensis strains

In a representative study, the MLVA-16 schedule was used to investigate molecular relationships between this study B. melitensis isolates and 296 B. melitensis isolates from other provinces of China. Five shared genotypes were observed in this population (Fig. 2; Additional file 5: Table S5), B. melitensis isolates from Guangxi Province had genotypes identical to those of strains from six different provinces, including the Inner Mongolia Autonomous Region, Shanxi, Henan, Shaanxi, Guangdong, and Zhejiang Provinces, and two out of five shared genotypes were comprised by isolates from Guangxi and Inner Mongolia Autonomous Region, 7 out of $16 \mathrm{~B}$. melitensis of this province had identical genotypes with strains from six provinces of China. Moreover, GX006 (Fig. 1, GT 26) had identical genotypes with strains from four different provinces, including Shaanxi (2011Jiang\#001) and Inner Mongolia (2011Jiang\#028, 2013Jiang\# 042), Henan (2011Jiang\#036), and Guangdong (2011Jiang\#027). Moreover, GX008 (Fig. 1, GT 23) obtained from blood samples of patients with brucellosis outbreaks in families in Hezhou City. Subsequently, scene epidemiology found that this family had a history of consuming raw ewe's milk. This strain had identical genotypes with strains from Inner Mongolia (2013)iang\#045).

\section{MLST genotyping}

All of isolates were further analyzed by MLST and three known MLST genotypes were identified: ST8 (3-2-3-2$1-5-3-8-2 ; \quad n=16), \quad$ ST17 (1-6-4-1-5-3-5-2-4; $n=18)$, ST21 (1-6-4-1-5-3-5-5-4; $n=6)$. Clustering analysis 


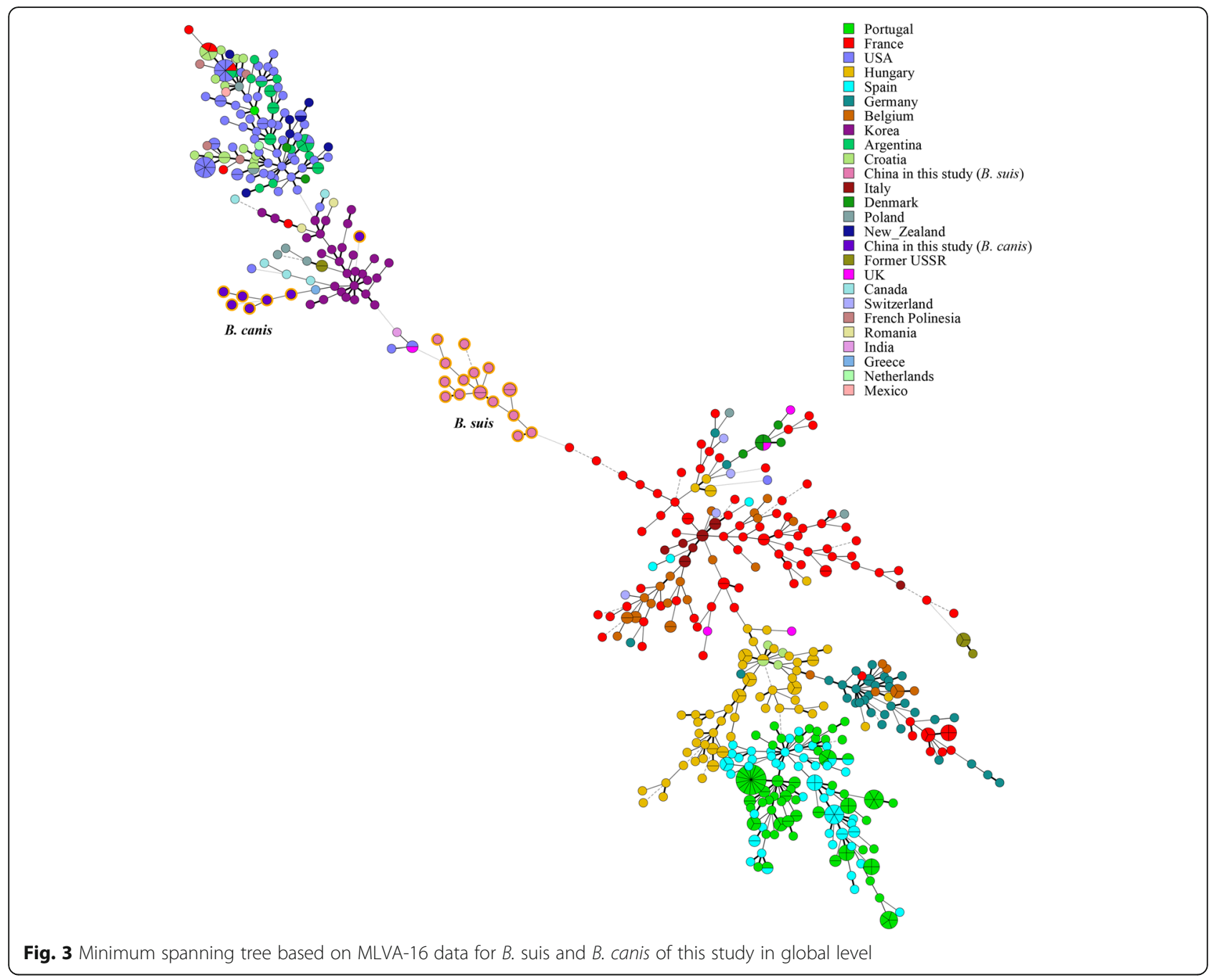

using BioNumerics software showed that the 40 isolates formed three main clusters (A C) (Fig. 4). All B. melitensis were distributed in cluster A and belong to genotype ST8. Cluster B including single genotype ST17 and all strains belonged to $B$. suis; cluster $C$ comprised $B$. canis and belonged to genotype ST21. These strains were divided into three distinct populations based on MLST typing data. There was a visible change in ST of strains isolated from 1961 to 2016 in this province; ST17 predominated among species before the 2000s, but ST8 was the most common population after the 2012. This assay is more suitable for discrimination at species levels and performed population construct investigation of Brucella isolates.

\section{Discussion}

In the present study, we employed both MLVA and MLST methods to characterize Brucella isolates were obtained from animals and humans in China's Guangxi Province from 1961 to 2016. Both methods showed an identical testing conclusion that there were at least three Brucella species in region examined. Biotyping identified 16 as B. melitensis biovar 3, 15 strains as $B$. suis biovar 3 and two as $B$. suis biovar 1 , and 7 strains as $B$. canis. Vaccination of breeding pigs with strain $\mathrm{S} 2$ has been the main strategy to control B. suis brucellosis in this province, but no $\mathrm{S} 2$ vaccine strain has been isolated in this study. Our survey showed that a total of 390,630 breeding pigs were vaccinated with strain S2 during 1996-1999, and 600, 000 breeding pigs were vaccinated in 2001-2002. The annual average vaccination coverage was higher than $80 \%$, which improved disease control. The prevalence rate of swine brucellosis in this province was $0.31 \%$ in $1996(36 / 11,521)$ and $0.00 \%(0 / 15,161)$ in 2004. Based on the time of isolation, B. suis biovar 3 was the predominant species in Guangxi before the 2000s, and only three cases of human brucellosis have been reported. Moreover, the circulating species have changed after 2012, with B. melitensis biovar 3 becoming 


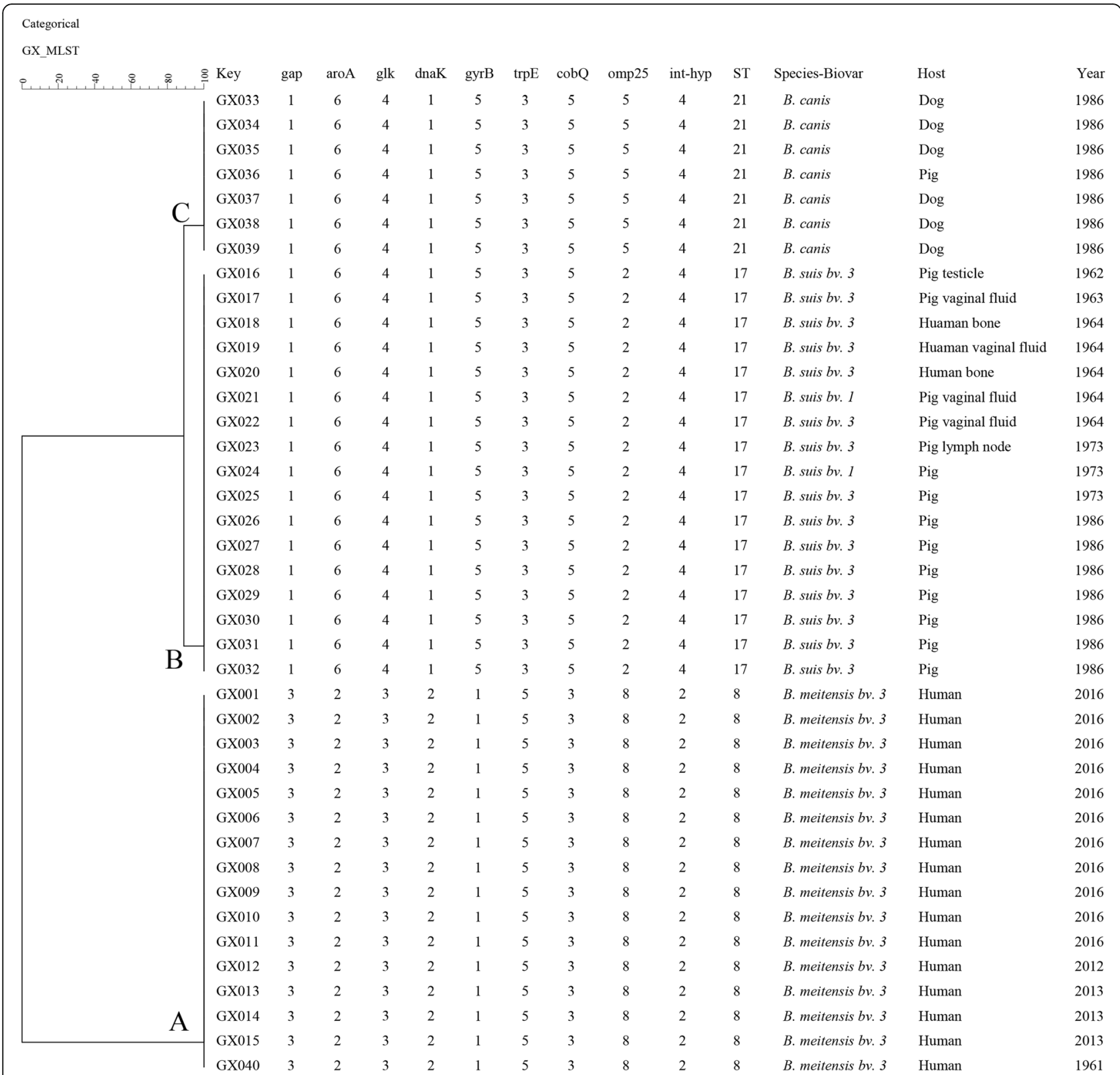

Fig. 4 Dendrogram based on the MLST genotyping assay showing relationships of 66 Brucella isolates. The columns show the identification numbers, ST types, species-biovar, host, and the year of isolation of the strains

the dominant species in this region, resulting in a dramatic increase in human brucellosis. Brucella isolates species/biovar typing confirmed that changes in dominant species is one of the main causes of a higher incidence rate of human brucellosis in this region [22]. Subsequently, all of strains were further examined by MLVA-16. Based on panel 1 markers, $B$. suis and $B$. canis all identified three new genotypes. However, none of the B. suis or B. canis panel 1 genotypes found in our study were identical to any of the genotypes in the Brucella 2016 MLVA database. These data indicated that although they had only just been reported, they may have been present historically. This conclusion was consistent with results of etiology analysis. B. melitensis was the predominant strain in most provinces, and B. suis in Guangxi was the dominant species of Brucella [9]. Panel 1 genotype 42 was the most common panel 1 genotype in China, mainly distributed in northeast and northwest of China. Moreover, in present study, $81.2 \%$ B. melitensis strains were found to belong to panel 1 genotype 42 , which was not reported in this area until 2012. This suggested it might be important to explore the molecular epidemiology underlying the higher 
incidence of human brucellosis in this region after 2012.

All B. melitensis strains were from the East Mediterranean group. Genotype 116 was the dominant population and distributed in northwestern China and worldwide in endemic areas of animal and human brucellosis [16], and responsibility for all human brucellosis cases after 2012 in Guangxi province. These data indicated that B. melitensis biovar 3 has recently come to circulate in this province. Moreover, Minimum Spanning Tree based on MLVA-16 analysis showed that there were five shared genotypes among $B$. melitensis strains in this study, and strains from other parts of China and the remaining $B$. melitensis were from a single genotype and very similar to strains from other provinces, suggesting that $B$. melitensis in this study may have originated in several different provinces. However, B. suis from this study formed a relatively independence branch and represent unique genetic lineage, which visibly diverged from strains from China and other nations; suggestion that epidemic of B. suis brucellosis was limited and sporadic cases are predominating. These population has characteristic of historical existence; it was exhibited potential trend for formed independence evolution branch. There was a very close relationship between B. canis strains from this study and those from Korea, which is very close to China geographically. The quarantine conditions set for allowing pet animals across national borders are relatively lacking [23].

Complete MLVA-16 locus yielded 34 genotypes and three main clusters (A-C). Cluster A comprised 15 Brucella strains were isolated after 2012, there is three MLVA16 genotypes were shared by two to three strains, and cluster rate was $44 \%(7 / 16)$. This information indicated that $44 \%(7 / 16)$ cases of this region had an outbreak epidemic profile after 2012, and other single genotypes were more sporadic. In addition, GX040 was isolated in 1961 and this strain exhibited unique genotypes when compared to isolates from other provinces in China. This showed that this strain may have been present historically and circulated only within a minor or definite range. Clusters B and C comprised 24 Brucella (B. suis and B. canis) strains isolated before the 2000s, of which four B. suis strains had two identical genotypes, suggesting that they had a common source of infection. Another 20 Brucella strains each represent single genotypes, indicating that $83.3 \%(20 / 24)$ Brucella (B. suis and $B$. canis) brucellosis had no epidemiological links or sporadic features, which was also a mainly brucellosis epidemic characteristics in Guangxi before 2012.

In a representative study, molecular epidemiological investigation of 312 Chinese $B$. melitensis strains were performed, showing extensive sharing of genotypes in this population (Fig. 2; Additional file 5: Table S5). B. melitensis isolates from Guangxi province had complete identical MLVA-16 genotypes with strains from six different provinces. Previous studies have confirmed that MLVA-16 genotyping results show good correlation with epidemiological data with epidemiologically related isolates displaying identical or very closely related genotypes [24, 25]. These data indicated that these strains may have molecular epidemiological links. Two out of five shared genotypes comprised isolates from Guangxi and the Inner Mongolia Autonomous Region. Seven out of 16 B. melitensis of this province had identical MLVA-16 genotypes with strains from five northwest provinces of China, suggesting that these regions were the most likely sources of infections for human brucellosis in Guangxi Province. This conclusion consistent with previous studies [26]. Brucellosis has been spreading from the northern provinces to the south. The proportion of imported brucellosis cases was higher in southern than in northern China after 2000 [11]. Furthermore, GX006 (Fig. 1, GT 26) had identical genotypes with simultaneous strains from four different northern provinces. These data hint that there are cases of crossinfections in these provinces and infected animals may help the contagion spread [27]. Moreover, GX008 (Fig. 1, GT 23) was obtained from blood samples of patients with brucellosis outbreaks in one family in Hezhou City, and this family had a history of consuming raw ewe's milk from a local farm that had a history of importing sheep northern China. Previous studies proved that consumption raw milk is also an important transmission route to human brucellosis [28]. To our surprised, this strain had identical MLVA-16 genotypes with strains from Inner Mongolia (2013)iang\#045). This suggested that the source of infections for this family of outbreaks was most probably Inner Mongolia. This is consistent with results from an epidemiological survey covering sheep imported from the northern provinces 6 months earlier. Strengthening quarantine inspections of animals crossing borders should become a priority.

MLST analysis revealed that 40 strains were divided into three distinct populations, ST8 $(n=16), \operatorname{ST} 17(n=$ $18)$, and ST21 $(n=6)$, respectively. ST17 and ST21 were the main populations in this province before 2000s. This was consistent with previous reports. Guangxi is the southernmost province in China, and it has been a site of brucellosis epidemics caused by $B$. suis. Mostly $B$. melitensis strains belonging to ST8 were found after 2012 and caused a sharp increase in the number of cases of human brucellosis. Moreover, ST8 was commonly distributed between northeastern and northwestern regions in China [21]. Consistent with previous reports, brucellosis caused by $B$. melitensis species has shown a visible trend toward geographic expansion from northern of China to southern provinces [26]. These observations inspired the hypothesis that this province experienced an epidemic of imported human brucellosis caused by $B$. melitensis biovar 3 from northwestern China. Moreover, a total of 3173 serum samples from 
sheep were assayed during 1996-2003, and none were positive. However, many aborted pregnancies in sheep were observed in this area examined in April 2004, and meanwhile, six positive sheep serum and two positive human serum samples were found. Since then, an epidemiological survey showed that introduction without quarantine from the northwest was the main cause for abortus events in sheep. The rate of human brucellosis was low at $0.35 \%$ during $1990-1999$, but increased to $7.01 \%(63 / 899)$ in 2004. At present, 624 human brucellosis cases have been reported during 2016-2018; these dates strongly support our hypothesis. However, due to imbalances and patchiness of the number of strains collected among different counties and among different years in this study, in-depth analyses of more Brucella strains are required to verify this conclusion and to elucidate the genetic and epidemiological characteristics of Brucella species in this province.

\section{Conclusion}

In this work, our team performed a comprehensive investigation of the molecular characteristics of Brucella species in Guangxi, China. There were at least three Brucella species, B. melitensis, B. suis, and B. canis, in this province. MLVA analysis showed extensive genotype sharing among $B$. melitensis from this study and other provinces within China. The B. melitensis isolated in this study may be a recent introduction into this area. B. suis formed an independent evolutionary branch and may have spread across a relative limited area. B. canis was very similar to strains from Korea. The previously predominant population, ST17, was replaced by the recent emerged ST8 group, which is the predominant ST in most provinces within China. Based on the results of a series of molecular investigations, we hypothesize that Guangxi experienced an imported human brucellosis epidemic caused by $B$. melitensis biovar 3, which might have come from northern China. We consider strengthening of inspections and quarantine of animals and animal products that cross borders imperative to control the imported human brucellosis. Our work provided important clues for enhancing the prevention and control of human and animal brucellosis in this region.

\section{Supplementary information}

Supplementary information accompanies this paper at https://doi.org/10. 1186/s12866-019-1665-6.

Additional file 1: Table S1. Table representing strain identification codes (Key), MLVA-8 data, MLVA-11 data, biovars, and year of isolation for 40 Brucella isolates.

Additional file 2: Table S2. 340 Chinese Brucella strains were used to construct the MST (strains in this study are indicated with yellow circles).
Additional file 3: Table S3. 615 Brucella strains (B. suis and B. canis) were used to construct the MST on a global scale (strains in this study are indicated with yellow circles).

Additional file 4: Table S4. Table representing isolates key, allele characters, ST, species, host, and year of collection of strains were used for MLST analysis.

Additional file 5: Table S5. Isolates from Guangxi province had identical MLVA-16 genotypes with strains from other provinces of China.

\section{Abbreviations}

MLST: Multilocus sequence typing; MLVA: Multiple locus variable-number tandem repeat analysis; PCR: Polymerase chain reaction; ST: Sequence type

\section{Acknowledgments}

We are grateful to all the staff in the Branch of Brucellosis Control and Prevention, Guangxi Province Centre for Disease Control and Prevention and for assistance in patient screening and patient epidemiology data collection. The authors would also like to thank LetPub (www.letpub.com) for their linguistic assistance during the preparation of this manuscript.

\section{Ethical approval and consent to participate}

This study is a retrospective molecular investigation of historically collected strains analyzed by modern typing methods. For this reason, the study did not involve the collection or reporting of patient data or any animal work, so we did not need to acquire consent to participate in this study.

\section{Authors' contributions}

LZG performed most of the strain isolations and MLVA typing. LZG also drafted the manuscript; WM performed the MLVA cluster analysis and MLST typing; ZHY was in charge of strains biotyping; PDR prepared the DNA samples; $\mathrm{JH}$ participated in the design of the study and critically reviewed the manuscript; LZJ participated in the design of the study and also managed the project. All authors read and approved the final manuscript.

\section{Funding}

This study was supported by National Key R\&D Program of China (Grant No. 2017YFC1200303, 2018ZX10734401, and 2018ZX10734404) and the Nature Science Fund of Inner Mongolia Autonomous Region (No. 2018MS08004). The funders had no role in study design, data collection and analysis, decision to publish, or preparation of the manuscript.

\section{Availability of data and materials}

All data generated or analyzed during this study are included in this published article and its supplementary information files, will be freely available to any scientist wishing to use them for non-commercial purposes upon request via e-mail with the corresponding author.

\section{Consent for publication}

Not applicable.

\section{Competing interests}

The authors declare that they have no competing interests.

\section{Author details}

${ }^{1}$ State Key Laboratory for Infectious Diseases Prevention and Control, National Institute for Communicable Disease Control and Prevention, Chinese Center for Disease Control and Prevention, 155 Changbai Road, Changping, Beijing 102206, People's Republic of China. ${ }^{2}$ Inner Mongolia Autonomous Region Comprehensive Center for Disease Control and Prevention, Huhhot 010031, People's Republic of China. ${ }^{3}$ Ulanqab Centre for Endemic Disease Prevention and Control, Jining 012000, Inner Mongolia, China.

Received: 10 January 2019 Accepted: 28 November 2019 Published online: 16 December 2019

\section{References}

1. Aghaali M, Mohebi S, Heydari H. Prevalence of Asymptomatic Brucellosis in Children 7 to 12 Years Old. Interdiscip Perspect Infect Dis. 2015;2015:187369. 
2. Ferreira AC, Chambel L, Tenreiro T, Cardoso R, Flor L, Dias IT, Pacheco T, Garin-Bastuji B, Le Fleche P, Vergnaud G, et al. MLVA16 typing of Portuguese human and animal Brucella melitensis and Brucella abortus isolates. PLoS One. 2012;7(8):e42514

3. Godfroid J, Garin-Bastuji B, Saegerman C, Blasco JM. Brucellosis in terrestrial wildlife. Rev Sci Tech. 2013;32(1):27-42.

4. Chen Y, Zhen Q, Wang Y, Xu J, Sun Y, Li T, Gao L, Guo F, Wang D, Yuan X, et al. Development of an extended multilocus sequence typing for genotyping of Brucella isolates. J Microbiol Methods. 2011;86(2):252-4.

5. Jiang H, Fan M, Chen J, Mi J, Yu R, Zhao H, Piao D, Ke C, Deng X, Tian G, et al. MLVA genotyping of Chinese human Brucella melitensis biovar 1, 2 and 3 isolates. BMC Microbiol. 2011;11:256.

6. Olsen SC, Palmer MV. Advancement of knowledge of Brucella over the past 50 years. Vet Pathol. 2014;51(6):1076-89.

7. Ran X, Cheng J, Wang M, Chen X, Wang H, Ge Y, Ni H, Zhang XX, Wen X. Brucellosis seroprevalence in dairy cattle in China during 2008-2018: A systematic review and meta-analysis. Acta Trop. 2019;189:117-23.

8. Harrison ER, Posada R. Brucellosis. Pediatr Rev. 2018;39(4):222-4.

9. Deqiu S, Donglou X, Jiming Y. Epidemiology and control of brucellosis in China. Vet Microbiol. 2002;90(1-4):165-82.

10. Liu ZG, Wang LJ, Piao DR, Wang M, Liu RH, Zhao HY, Cui BY, Jiang H. Molecular Investigation of the Transmission Pattern of Brucella suis 3 From Inner Mongolia, China. Front Vet Sci. 2018:5:271.

11. Lai S, Zhou H, Xiong W, Gilbert M, Huang Z, Yu J, Yin W, Wang L, Chen Q, Li Y, et al. Changing Epidemiology of Human Brucellosis, China, 1955-2014. Emerg Infect Dis. 2017;23(2):184-94

12. Hanot Mambres D, Boarbi S, Michel P, Bouker N, Escobar-Calle L, Desqueper D, Fancello T, Van Esbroeck M, Godfroid J, Fretin D, et al. Imported human brucellosis in Belgium: Bio and molecular typing of bacterial isolates, 19962015. PLoS One. 2017;12(4):e0174756.

13. Le Fleche P, Jacques I, Grayon M, Al Dahouk S, Bouchon P, Denoeud F, Nockler K, Neubauer H, Guilloteau LA, Vergnaud G. Evaluation and selection of tandem repeat loci for a Brucella MLVA typing assay. BMC Microbiol. 2006;6:9.

14. Piao DR, Liu X, Di DD, Xiao P, Zhao ZZ, Xu LQ, Tian GZ, Zhao HY, Fan WX Cui BY, et al. Genetic polymorphisms identify in species/biovars of Brucella isolated in China between 1953 and 2013 by MLST. BMC Microbiol. 2018; 18(1):7.

15. Kong W. Brucellosis infection increasing in Southern China. Eur J Intern Med. 2018;51:e16-8

16. Cao X, Li Z, Liu Z, Fu B, Liu Y, Shang Y, Zhou J, Jing Z. Molecular epidemiological characterization of Brucella isolates from sheep and yaks in northwest China. Transbound Emerg Dis. 2018;65(2):e425-33.

17. Al Dahouk S, Tomaso H, Nockler K, Neubauer H, Frangoulidis D. Laboratorybased diagnosis of brucellosis--a review of the literature. Part I: Techniques for direct detection and identification of Brucella spp. Clin Lab. 2003;49(910):487-505.

18. Al Dahouk S, Fleche PL, Nockler K, Jacques I, Grayon M, Scholz HC, Tomaso $H$, Vergnaud G, Neubauer H. Evaluation of Brucella MLVA typing for human brucellosis. J Microbiol Methods. 2007;69(1):137-45.

19. Liu ZG, Di DD, Wang M, Liu RH, Zhao HY, Piao DR, Tian GZ, Fan WX, Jiang H, Cui BY, et al. MLVA Genotyping Characteristics of Human Brucella melitensis Isolated from Ulanqab of Inner Mongolia, China. Front Microbiol. 2017:8:6.

20. Whatmore AM, Perrett LL, MacMillan AP. Characterisation of the genetic diversity of Brucella by multilocus sequencing. BMC Microbiol. 2007;7:34

21. Cao X, Shang Y, Liu Y, Li Z, Jing Z. Genetic Characterization of Animal Brucella Isolates from Northwest Region in China. Biomed Res Int. 2018; 2018:2186027.

22. Chen $Y$, Ke $Y$, Wang $Y$, Yuan $X$, Zhou $X$, Jiang $H$, Wang $Z$, Zhen $Q$, Yu $Y$, Huang $L$, et al. Changes of predominant species/biovars and sequence types of Brucella isolates, Inner Mongolia, China. BMC Infect Dis. 2013;13:514.

23. Kang SI, Heo EJ, Cho D, Kim JW, Kim JY, Jung SC, Her M. Genetic comparison of Brucella canis isolates by the MLVA assay in South Korea. J Vet Med Sci. 2011;73(6):779-86

24. Tay BY, Ahmad N, Hashim R, Mohamed Zahidi J, Thong KL, Koh XP, Mohd Noor A. Multiple-locus variable-number tandem-repeat analysis (MLVA) genotyping of human Brucella isolates in Malaysia. BMC Infect Dis. 2015;15:220

25. Kilic S, Ivanov IN, Durmaz R, Bayraktar MR, Ayaslioglu E, Uyanik MH, Aliskan H, Yasar E, Bayramoglu G, Arslanturk A, et al. Multiple-locus variable-number tandem-repeat analysis genotyping of human Brucella isolates from Turkey. J Clin Microbiol. 2011;49(9):3276-83.

26. Tian GZ, Cui BY, Piao DR, Zhao HY, Li LY, Liu X, Xiao P, Zhao ZZ, Xu LQ, Jiang H, et al. Multi-locus variable-number tandem repeat analysis of Chinese Brucella strains isolated from 1953 to 2013. Infect Dis Poverty. 2017;6(1):89.

27. Shevtsov A, Ramanculov E, Shevtsova E, Kairzhanova A, Tarlykov P, Filipenko M, Dymova M, Abisheva G, Jailbekova A, Kamalova D, et al. Genetic diversity of Brucella abortus and Brucella melitensis in Kazakhstan using MLVA-16. Infect Genet Evol. 2015;34:173-80.

28. Mugizi DR, Muradrasoli S, Boqvist S, Erume J, Nasinyama GW, Waiswa C, Mboowa G, Klint M, Magnusson U. Isolation and molecular characterization of Brucella isolates in cattle milk in Uganda. Biomed Res Int. 2015;2015:720413.

\section{Publisher's Note}

Springer Nature remains neutral with regard to jurisdictional claims in published maps and institutional affiliations.
Ready to submit your research? Choose BMC and benefit from:

- fast, convenient online submission

- thorough peer review by experienced researchers in your field

- rapid publication on acceptance

- support for research data, including large and complex data types

- gold Open Access which fosters wider collaboration and increased citations

- maximum visibility for your research: over $100 \mathrm{M}$ website views per year

At BMC, research is always in progress.

Learn more biomedcentral.com/submissions 